\title{
A Class Act - revising food classifications to enable automated assessment of compliance with food-based dietary guidelines
}

\author{
H. Al-Sehaim ${ }^{1,2}$ and F. E. Douglas ${ }^{1}$ \\ ${ }^{1}$ Nutritics, Nutrition Analysis Software, Town Plaza, Swords, Co. Dublin and \\ ${ }^{2}$ School of Biological and Health Sciences, Technological University Dublin, Kevin Street, Dublin 8
}

Food classification systems make it easier to compare data from various sources and aids in the creation of public health guidelines. These systems can include a large amount of foods divided into various food groups and broader food categories. ${ }^{(1)}$ Nutritics employs a standardised food categorisation system containing 114 unique categories. Food-based dietary guidelines (FBDG) categorise food types into general food groups to enable communication around amounts to consume daily basis to promote good health. ${ }^{(2)}$ The correct classification of foods is essential to enable the implementation of an automated feature in Nutritics to assess compliance with FBDGs.This project aims to revise Nutritics categories associated with foods from the McCance and Widdowson's Composition of Food Integrated Dataset (CoFID) - the food composition data applicable for use in the UK and Ireland. Also, to quantify the number of foods in each unique category and to calculate the quantity of foods that require a category update.

The CoFID dataset, containing a total of 3,291 foods, was revised. Foods were assigned an appropriate category from one of the 114 Nutritics categories or remained in their original category. The number of foods in each Nutritics category originally and the number of foods in each new Nutritics category were recorded and compared to determine the total number of foods that required an update. Similar food categories were grouped together for analysis. Alcoholic beverages and baby foods were excluded from this study.

A total of 3,237 foods and beverages from the dataset were analysed across 94 unique Nutritics categories. A total of 77 categories out of 94 had foods that required a category update $(82 \%)$. The total number of foods that needed a category update was $1,045(32 \%)$. The food category that needed the largest number of updates was the Vegetables-General group $(n=144)$. Thirteen categories contained only 1 food that required re-classification. The mean number of foods that needed a category update were calculated for each category grouping as follows: Protein categories $(\mathrm{n}=27), 7(0 \mathrm{~min}-32 \mathrm{max})$; Starchy categories $(\mathrm{n}=14), 6(0 \mathrm{~min}-22 \mathrm{max})$; Fruit and Vegetable categories $(n=10), 44(4 \min -144 \max )$; Dairy and Alternative categories $(n=9), 6(0$ min -18 max); Other Dishes and Snacks categories $(n=6), 22(0$ min - 117 max); Desserts and Treats $(n=7), 16(\min 0 / \max 85)$; Beverage categories $(n=10), 2$ $(\min 0 / \max 5)$; Miscellaneous categories $(\mathrm{n}=11) 2(0 \min -\max 6)$.

The optimisation of foods assigned to a Nutritics category is necessary to enable appropriate rules to be applied to foods at a category level. Nutritics will use this optimised data to implement a feature to support users to automatically assess compliance with food group targets defined by FBDGs.

1. European Food Safety Authority (2015) [Available at: https://efsa.onlinelibrary.wiley.com/doi/pdf/10.2903/sp.efsa.2015.EN-804]

2. Herforth, A., Arimond, M., Álvarez-Sánchez, C., et al. (2019) Advances in Nutrition 10, 4, 590-605 